\title{
Kinetics and mechanism of the adsorption of methylene blue from aqueous solution on the surface of a quartz cuvette by on-line UV-Vis spectrophotometry
}

\author{
József Kalmár ${ }^{\mathrm{a} *}$, Gábor Lente ${ }^{\mathrm{b}}$ and István Fábián ${ }^{\mathrm{b}}$ \\ a MTA-DE Homogeneous Catalysis and Reaction Mechanisms Research Group, \\ Egyetem tér 1, H-4032 Hungary \\ ${ }^{\mathrm{b}}$ Department of Inorganic and Analytical Chemistry, University of Debrecen, \\ Egyetem tér 1, H-4032 Hungary \\ *Author of correspondence: e-mail: kalmar.jozsef@science.unideb.hu
}

\begin{abstract}
It is often challenging to follow fast surface adsorption processes with sufficient time resolution. Here we report a convenient UV-Vis spectrophotometry based method to study the fast kinetics of anarchetypicalsurface process:the adsorption ofMBonquartz. The test object was a commercial quartz cuvette.About $3.3 \%$ of the dyewasadsorbed from a $34 \mu \mathrm{M}$ solution at $\mathrm{pH} 7$ in $10 \mathrm{~min}$. Biphasic kinetics was observed for the depletion of MB under continuous stirring at $1000 \mathrm{rpm}$. A kinetic model was postulated for the interpretation of time resolved spectral changes.This model assumes the reversible adsorption of MB on the surface which is followed by the isomerizationof the binding mode. The overall process is kinetically first order with respect to $\mathrm{MB}$, thus dimer formation, which waspreviously proposed to be favored on quartz, can be ruled out. The experimental adsorption isotherm is consistent with the formation of an adsorption monolayer from single molecules. The UV-vis absorbance spectrum of adsorbed MB does not show any blue shift.With the simplest experimental setup, it is shown that the dimerization of $\mathrm{MB}$ is not exceptionally favored on quartz surface.
\end{abstract}

Keywords.methylene blue, quartz, adsorption, kinetics, mechanism 


\section{Introduction}

Methylene blue (MB) is often a model compound in adsorption studies. Due toits planar structure with 14 delocalized electrons, this compound is easily involved in stacking interactions. Consequently, MB readily forms dimers and higher level aggregates in solution even at micro-molar analytical concentrations. [1,2,3]The interplay of stacking interactions and Coulomb forces governs the adsorptive properties of this cationic dye. [4]Thus, hydrophilic surfaces with net negative charges also attract MB. Consequently,MB readily adsorbs onto a wide variety of adsorbents from industrial wastes[5]to advanced materials $[6,7,8]$ including (modified) minerals. [9,10]

The adsorption of MB on cheap industrial waste products is frequentlystudied to gain information regarding the removal of pollutants from wastewater. Simplified evaluation methods are often used for the interpretation of the kinetic and thermodynamic results in these studies.[11]The adsorption of MBon advanced materials is typically characterized by spectroscopic methods, as well as direct thermodynamic measurements in order to explore the binding mode and the orientation of the molecule on the surface. [12,13,14]

The thermodynamic and UV-Vis spectroscopic features of $\mathrm{MB}$ adsorbed on silica surfacearewell characterized. $[13,14,15,16,17]$ There seems to be a consensus in the literature that $\mathrm{MB}$ forms a monolayer on silica when adsorbed from solutions of high micromolar concentrationsat short contact times. The UV-Vis spectrum of adsorbed MB shows a blue shift at around $600 \mathrm{~nm}$, which has beenattributed to dimer formation on the surface. This hypothesis is based on a similar blue shift in the spectrum of solvated dimers. [18]The fast adsorption kinetics of MB on silica has not been studied before. Only a handful of studies with high time resolution (seconds) were reported on the adsorption kinetics of proteins $[19,20]$ and simple inorganic ions. [21]The common feature of these studies is the use of special detectors, which can produce a response fast enough to follow the rapid layer formation on the surface probes.

Inour experimental work, we observed an about $1-3 \%$ UV-Vis absorbance decay when an aqueous solution of $\mathrm{MB}$ was introduced into a $1.00 \mathrm{~cm}$ quartz cuvette. By using this simple experimental setup, we designed and conducted a kinetic study with multi-wavelength spectrophotometric detection to explore the mechanism of the adsorption process.Unexpectedly, we found, that dimerization of MB is not favored on a quartz surface. 


\section{Experimental}

2.1. Chemicals and solutions. Methylene blue hydrate (purum p.a., 97+\%), $\mathrm{NaH}_{2} \mathrm{PO}_{4} \cdot \mathrm{H}_{2} \mathrm{O}$ (ACS reagent, 98+\%), $\mathrm{NaOH}$ (reagent grade, 98+\%) and 70\% $\mathrm{HClO}_{4}$ (ACS reagent)were purchased from Sigma-Aldrich (St. Louis, MO, USA). Ion exchanged and Milli-Q Synthesis A10ultra-filtered (EMD Millipore, Billerica, MA, USA) water was used to prepare all solutions. The resistivity of this water was never smaller than18.2 M $\Omega$, and ithadno detectable organic content at the level of $>10 \mathrm{ppb}$. This water is referred to as DI water in the rest of this paper.pH was measured by a Metrohm 6.0234.110 combined glass electrode attached to a Metrohm 721 NET Titrino titrating unit. The electrode was calibrated by two buffers according to IUPAC recommendations. [22]The ionic strength was adjusted and kept at a fixed value in all experiments by usingNaH $\mathrm{PO}_{4}-\mathrm{Na}_{2} \mathrm{HPO}_{4} 25 \mathrm{mM}-25 \mathrm{mM}$ buffer $(I=0.10$ $\mathrm{M})$.

2.2. Quartz cuvettes.Hellma 110-QS quartz cuvettes of $10.00 \mathrm{~mm}$ optical pathlength and $3500 \mu \mathrm{L}$ total volume were exposedto methylene blue solutions to test the adsorption of the dye onto their inner surface. These cuvettesare manufactured from synthetic quartz of the highest purity and homogeneity according to the manufacturer.Four cuvettes were tested with different batch numbers. The cuvettes were soaked in chromosulfuric acid for 1 day upon arrival from the manufacturer. After soaking, the cuvetteswere washed with Milli-Q water, then with 1:1 HCl, and finally with Milli-Q water. Thisextracleaning procedure was repeated every 3 weeksin addition to the regular cleaning process between the experiments (as described in the section 'Adsorption measurements' later).Brand new cuvettes were used in this study, i.e. they were never used for any other experiment in order to avoid crosscontamination.

2.3. Instruments.UV-vis absorbance was measured at multiple wavelengths simultaneously in a custom built spectrophotometer equipped with an AvaSpec-ULS-2048LTRC CCD detector manufactured by Avantes (Apeldoorn, The Netherlands). This spectrophotometer is part of a custom built photoreactor.A similar device is already described in the literature. [23] Our detector is typically operated with $15 \mu$ s integration time and $20-50$ subsequent spectra in the 200-1000 $\mathrm{nm}$ wavelength rangeare averaged for smoothing. The cell holder of this instrument is equipped with a Peltierthermostat unit and a built-in magnetic stirrer. UV- 
vis spectra were also recorded in a PerkinElmer Lambda 25 scanning spectrophotometer (PerkinElmer, Waltham, MA, USA).

2.4. Adsorption measurements. The most convenient way to study the adsorption of MBon the inner surface of aquartz cuvette isby simply injecting an aqueous MB solution into an empty cuvette and following the spectral change on-line by a UV-vis spectrophotometerunder continuous stirring. However, we experienced better reproducibility and robustnesswhen the MB solution was not directly injected intothe empty cuvette, but into an aqueous buffer already stirred insidethe cuvette, whichresulted in an in situdilution of MB. The drawback of this method is thatsome timeis required forcomplete homogenization. We used the following general experimental protocol.The quartz cuvette was thoroughly washed with $1: 1 \mathrm{HCl}$ and DI water, and equilibrated with the buffer for several minutes between each run. Thecuvette containing $1.5-2.0 \mathrm{~mL}$ aqueous phosphate bufferwas placed into the cell holder of the spectrophotometer, thermostated to $25^{\circ} \mathrm{C}$ and stirred by a Teflon coated $2 \times 8 \mathrm{~mm}$ magnetic rod at $1000 \mathrm{rpm}$. Spectrophotometric detection was started, anda few seconds later,10 $500 \mu \mathrm{L}$ buffered MB solution was promptly injected into the cuvette by a pipette.Absorbance change was followed in the 200 - 800 nmwavelength range for at least $600 \mathrm{~s}$ with a minimum time resolutionof $1 \mathrm{~s}$. The moment of injection can clearly be identified in the recorded kinetic traces.Thismethod was validated and the time needed for complete homogenizationduringinsitu mixing was determined by comparing experimentalkinetic curvesrecorded with or without using a diluentunder otherwise identical conditions(Figure 1). The time scale of the kinetic curve recordedby filling an empty cuvettewith dilute MB solutionhas to be shifted by $8 \mathrm{~s}$ to match the kinetic curve recorded using in situ dilution. Thus, we concluded thatcomplete homogenization during insitu mixingis achieved within $8 \mathrm{~s}$ after injecting a concentrated MB solution into the diluent.

In the experimentsdescribed above, steadyabsorbance decay was detected, whichwasattributed exclusively to the depletion of the dye from solutiondue to adsorption on quartz surface, as detailed further in the paper. 


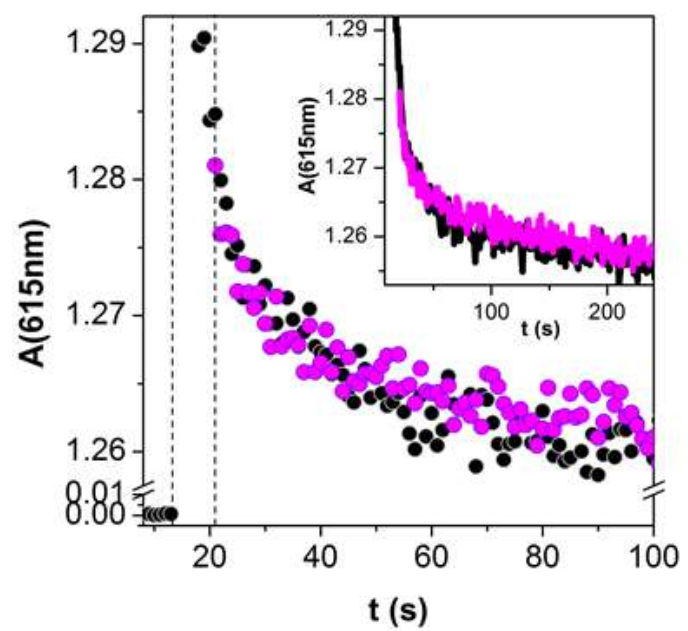

Figure 1.Absorbance change detectedafterinjecting a methylene blue (MB) solution into a quartz cuvette. The first kinetic curve (black points) was recorded by injecting $70 \mu \mathrm{L}$ of a $1.00 \mathrm{mM} \mathrm{MB}$ solution into $2.00 \mathrm{~mL}$ buffer stirred in a cuvette, thus insitu diluting MB. In this case,some timeis required for complete homogenization.The second curve (magenta points) was recorded afterinjecting $2.00 \mathrm{~mL}$ of a33.8 $\mu \mathrm{M}$ MB solution into an empty dry cuvette containing only a stirrer. The first vertical dotted line at $13 \mathrm{~s}$ indicates the time of injection of the dye in the first experiment. The second dotted line at $21 \mathrm{~s}$ indicates the time of injection in the second experiment. The timeshift between the two kinetic curves, which gives the mixing time (i.e. the time needed for complete homogenization) in the first experimentis $8 \mathrm{~s}$. The timeshift requiredfor the two kinetic curves to match was found by manually moving the second curve along the $x$-axis. The inset shows the same two kinetic curveson a longer time scale. $c_{0}(\mathrm{MB})=33.8 \mu \mathrm{M} ; c\left(\mathrm{NaH}_{2} \mathrm{PO}_{4}+\mathrm{Na}_{2} \mathrm{HPO}_{4}\right)=50 \mathrm{mM} ; \mathrm{pH}=6.84 ; 25^{\circ} \mathrm{C} ; 1000 \mathrm{rpm}$ mixing.

2.5. Data treatment. The experimental data were fitted to the appropriate expressions using Levenberg-Marquardt leastsquares procedures with theMicromath Scientist 2.0 software (Micromath Scientific Software, St. Louis, MO, USA). Simultaneous non-linear fitting of multiple kinetic curves recorded under different initial conditions (global kinetic analysis) was performed with the program package ZiTa [24]using the GEAR algorithm. [25] Multiwavelength kinetic data were evaluated with the SpecFit/32 software (Spectrum Software Associates, Chapel Hill, NC, USA). 


\section{Results}

3.1. Solution phase dimerization of methylene blue.The UV-Vis spectraof aqueous MB solutionswere recorded in the concentration range from $1.00 \mu \mathrm{M}$ to $1.00 \mathrm{mM}$ in cuvettes of various opticalpathlengths. The absorbance versusc $(\mathrm{MB})$ plots are non-linear atvarious wavelengths (Table S1 and Fig. S4 in the Supporting Information). This phenomenon can be unambiguously attributed to the solution phase dimerization of MB.[3] Evaluation of the spectrophotometric data yielded $\log \left(K_{\mathrm{f}}\right)=3.9 \pm 0.1$ for the dimerization equilibrium constant of $\mathrm{MB}$ in phosphate buffer at $\mathrm{pH} 6.9, I=0.10$.This result is in reasonable agreement with literature data, which were reported $\log \left(K_{\mathrm{f}}\right)$ values between 3.5 and 3.9 under different conditions. [2,3] Details of the evaluation are discussed in the Supporting Information.

3.2. Adsorption of methylene blue.The main goals of the first setsof experiments wereto test reproducibility and to check whether the absorbance decay shown in Figure 1can exclusively be attributed to the adsorption of the dye after the injection of a MB solution into a quartz cuvette. All the common possibilities (adsorption, contamination, shifting of the solution phase dimerization equilibrium, redox reaction)were considered, and everything but adsorption was ruled out on the basis of argumentsgiven in the Discussion.

Stirring has a remarkable effect on the rate of absorbance change.The absorbance of MB decreases slowlywithout stirring. However, turning thestirring on causes a relatively fast drop in the measured absorbance(Figure S5 in the Supporting Information). Constant stirring at $1000 \mathrm{rpm}$ ensured excellent reproducibility.

Adsorption experiments were repeated by mixing $\mathrm{MB}$ solutions of different concentrations with appropriate buffer solutions in a way that the final samples were identical. The excellent reproducibility of the kinetic traces provesthe robustness of our experimental protocol (Figs. S6 and S7). These results also confirm that partial temporal inhomogeneity does notinterfere with the absorbance measurement. Neither the kinetics nor the amplitude of the observed absorbance change is altered when the concentration of theinjected $\mathrm{MB}$ solutionand, as a consequence, the magnitude of in situ dilution are changed in these experiments. Since the extent of dimerization depends on the initial concentration of MB, these observations also imply that shiftingof the solution phase dimerization equilibrium does not have any effect on the adsorption of MB on quartz surface.

The effect of $\mathrm{pH}$ on theadsorption of MB was studied in 3 sets of experiments at $\mathrm{pH} 2,7$ and 12. Only a minor absorbance change was observed at $\mathrm{pH} 2$ where MB is protonated(Figs. 
S8 and S9). This absorbance changeis smaller than in neutral and alkaline solutions. One explanation is the decrease of the molar absorbance of $\mathrm{MB}$ due to protonation.However, themolar absorbances are about the same at $\mathrm{pH} 7$ and 12, and the larger absorbance change indicates more pronounced adsorption at higher $\mathrm{pH}$. Thisobservation strongly suggests that the protonation of $\mathrm{MB}$ and the quartz surface makes the adsorption of the dye less favorable.

The reversibility of the adsorption of MB was also tested. For this purpose, a cuvette was pretreated with MB solution overnight. After removing this solution, phosphate buffer was added to the empty cuvette and stirred. Increasing absorbance clearly confirmed desorption of $\mathrm{MB}$ from the quartz surface.Details are given in the Supporting Information (Figs.S10 and S11).

The efficiency of the cleaning protocol used for cleansing the cuvettes was also tested.One cuvette was soaked in "piranha solution" (containing peroxomonosulfuric acid, a.k.a. Caro's acid, $\mathrm{H}_{2} \mathrm{SO}_{5}$ ) for $2 \mathrm{~h}$, and another cuvette was soaked in double-distilled acetone overnight before an adsorption experiment. The kinetic curves recorded with these cuvettes were identical with those obtained in cuvettes cleanedby chromosulfuric acid and 1:1 $\mathrm{HCl}$ between experimental runs. Thus, memory effects or contamination on the surface could not falsify our experimental results.

We also made an effort to prove that the adsorption phenomenon is associated with the specific natural features of the quartz surface. A number ofpractical methods are known for making glass and quartz laboratory equipment inert against the adsorption of ions. $[26,27]$ Treatment of the cuvette with a solution of dichloro-dimethylsilane in hexane for several hours made the quartz surface less susceptible toward the adsorption of the cationic $\mathrm{MB}(c f$.Fig. S12). Presumably, this phenomenon is the consequence of the methylation of Si$\mathrm{OH}$ groups on the surface, which in turn becomes less capable to bond cationic MB.

3.3. Kinetics of adsorption of methylene blue.Kinetic experiments were performed in order to study the mechanism of the adsorption of methylene blue (MB) on the surface of a quartz cuvette at pH 6.9 in $50 \mathrm{mM}$ phosphate buffer $(I=0.10 \mathrm{M})$. These experiments were carried outfollowing the general protocol described in the Experimental section. The initial concentration of MB was varied between5.0and $34 \mu \mathrm{M}$. After injecting a MB solution into a buffer solution, a steady decrease in absorbance was detected at all wavelengths with biphasic kinetic characteristics. The first kinetic step is complete in ca. $30 \mathrm{~s}$ and the second step takesabout 300 s. Sample kinetic curves areshown in Fig. 2. 


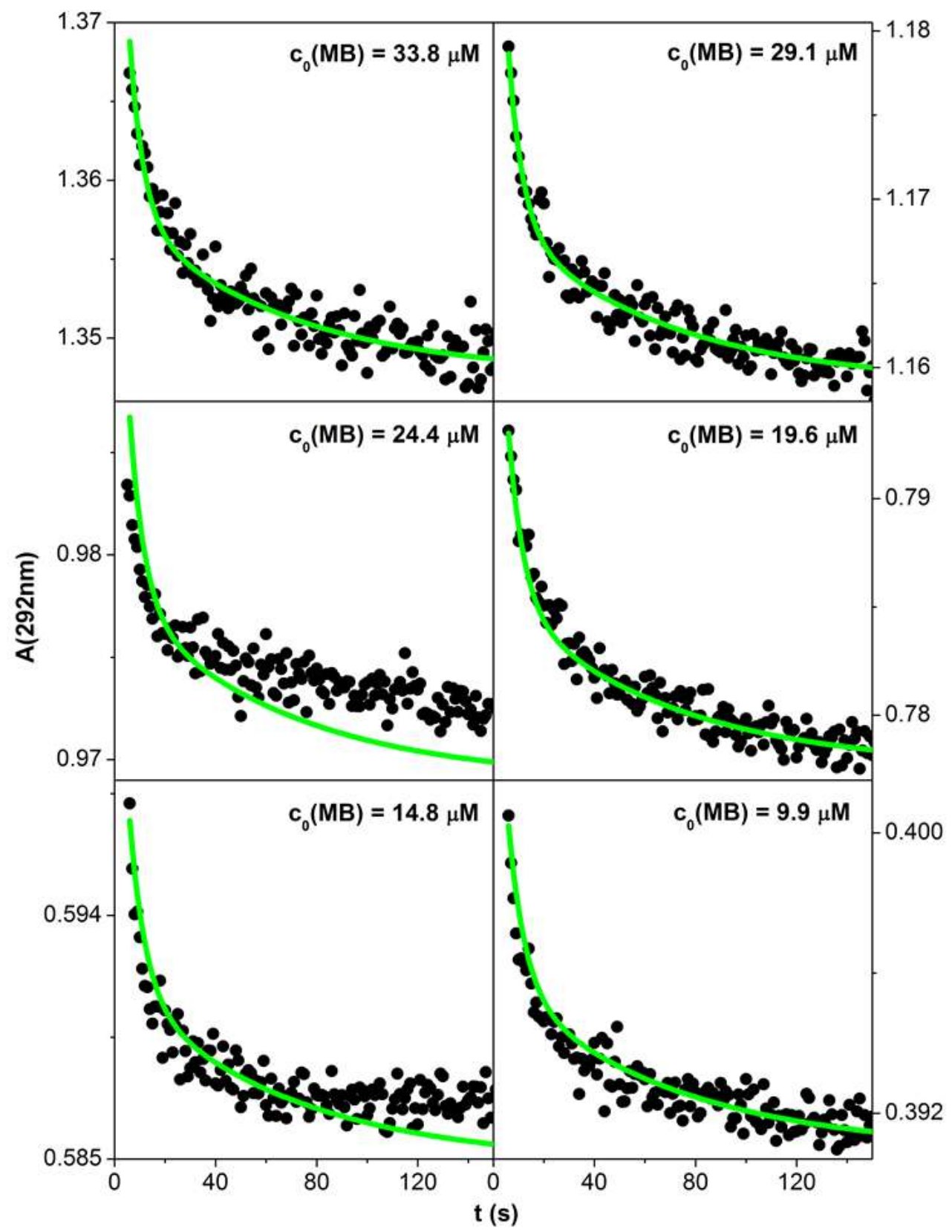

Figure 2. Kinetic curves detected afterinjecting $10-70 \mu \mathrm{L}$ of a $1.00 \mathrm{mM}$ MB solution in phosphate buffer into $2.00 \mathrm{~mL}$ buffer solution stirred in a quartz cuvette. Kinetic curves are shown from $8 \mathrm{~s}$ after injection. Black dots are experimental data points. Continuous green lines are the results of global data fitting to the kinetic model given inScheme 1(describing the adsorption of MB on quartz surface). Two kinetic curves that were also included in the analysis $\left(c_{0}(\mathrm{MB})=7.4 \mu \mathrm{M}\right.$ and $\left.5.0 \mu \mathrm{M}\right)$ are not shown.c $\left(\mathrm{NaH}_{2} \mathrm{PO}_{4}+\mathrm{Na}_{2} \mathrm{HPO}_{4}\right)=50 \mathrm{mM} ; \mathrm{pH}=6.89 ; 25^{\circ} \mathrm{C} ; 1000 \mathrm{rpm}$ mixing.

Characteristic spectral changes recorded in the kinetic experiments are shown as difference spectra in Fig. 3. (The primer spectra are shown in Fig. S13 in the SI.) The number of the light absorbing components in a time-resolved UV-Vis spectral series can be estimated by the matrix rank analysis or by the singular value decomposition analysis of the 
experimental data. $[28,29,30]$ Our multi-wavelength data sets are badly conditioned for these mathematical methods becausethe relative change in absorbance is not more than $3 \%$ over the whole wavelength range. Nevertheless, the analysis of time resolved spectra at different initial MB concentrations predicts that the number of significant singular values is 2 (cf. Table S2 in theSupporting Information). The results are consistent withthe presence of the main absorbing species (hydrated MB) and an additional weaklyabsorbing component(presumably the absorbed MB, $c f$. Discussion). Plotting absorbance values recorded in the same experiment at different wavelengths versus each other always givesa straight line regardless of the chosen wavelength pairs (an example is shown in Fig. S14 in the SI). This proportionality meansthat the data obtained at different wavelengths are highly correlated, i.e., the kinetic trace at each wavelength carries the same kinetic information.

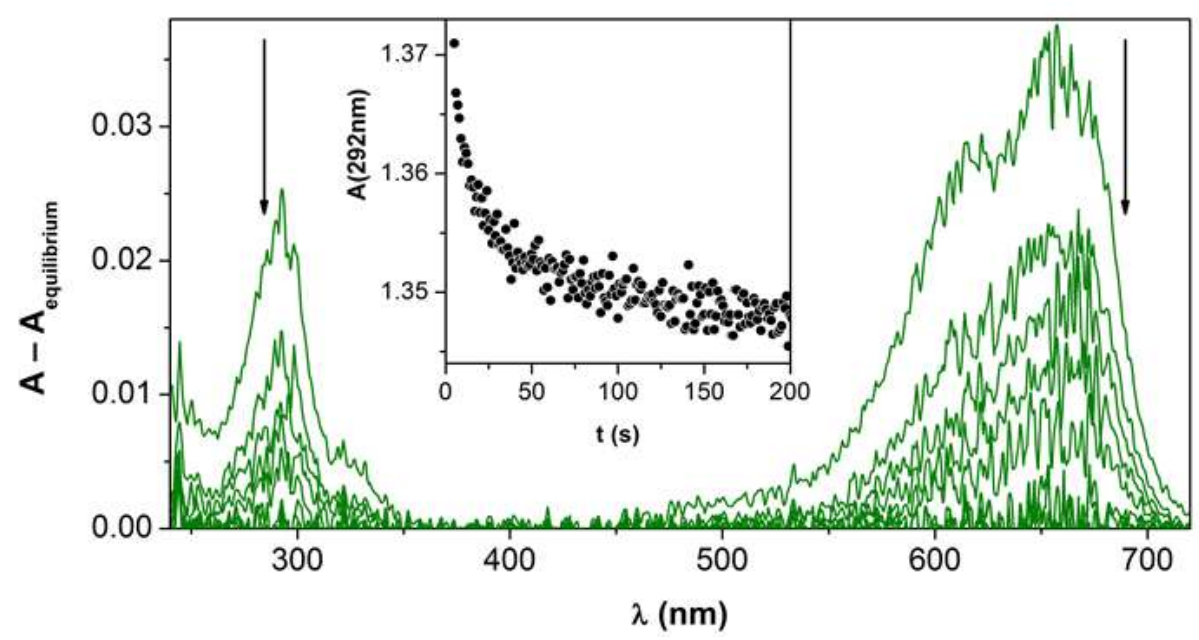

Figure 3.Time resolved difference spectra obtainedafterinjecting $70 \mu \mathrm{L}$ of a $1.00 \mathrm{mM}$ methylene blue (MB) solution into $2.00 \mathrm{~mL}$ buffer stirred in a quartz cuvette. The spectra were calculated by subtracting the final spectrum (the spectrum recorded when the adsorption process was complete) from the spectrum recorded at a given reaction time.Spectraare shown from $8 \mathrm{~s}$ to 200 safter injection. Only $2 \%$ of the measured spectra are shown for clarity of presentation.Inset: the corresponding kinetic curve at $292 \mathrm{~nm}$. The primer spectra are shown in Fig. S13 in the SI.

$\mathrm{c}_{0}(\mathrm{MB})=33.8 \mu \mathrm{M} ; \mathrm{c}\left(\mathrm{NaH}_{2} \mathrm{PO}_{4}+\mathrm{Na}_{2} \mathrm{HPO}_{4}\right)=50 \mathrm{mM} ; \mathrm{pH}=6.89 ; 25^{\circ} \mathrm{C} ; 1000 \mathrm{rpm}$ mixing.

The transmission UV-Vis spectrum of MB adsorbed on the wall of the cuvette was also recorded as follows. First, the cuvette was equilibrated for 15 min with a MB solution in phosphate buffer, then it was flushed with a few drops of water and left dripping for 5 min. The spectrum of this empty pre-treated cuvette represents the UV-Vis spectrum of MB 
adsorbed on its quartz wall. This experiment was performed at several MB concentrations, and we found that the spectrum of adsorbed MB does not show any blue shift compared to its solution phase spectrum when $10-50 \mu \mathrm{M}$ solutions were used for equilibration. However, the spectrum of adsorbed MB shows a significant blue shift when the cuvette is treated with a $1.0 \mathrm{mM}$ MB solution (Fig. 4). The similarity of the spectra of adsorbed and solution phase $\mathrm{MB}$ at low concentrationsindicates that MB probably forms a monolayer onthe quartz surface in the applied concentration range. Aggregates are not formed when the cuvette is treated with relatively dilute MB solutions. Thus, it is also reasonable to assume that the dimerization of $\mathrm{MB}$ is not favored on quartz. Apparently, dimerization on the surfacetakes place only when the surface is in contact with more concentrated $(100-1000 \mu \mathrm{M})$ MBsolutions, which already contain MB dimer in substantial concentrations.

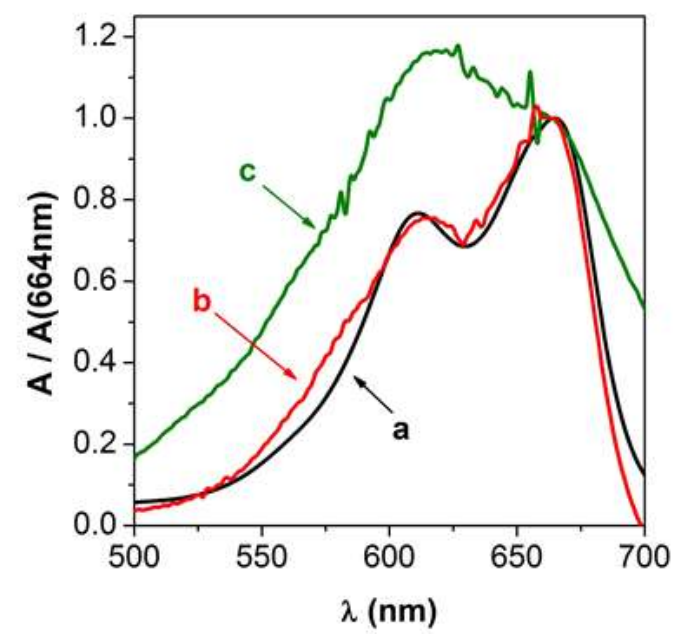

Figure 4. a) UV-Vis spectrum of a $50 \mu \mathrm{M}$ aqueous MB solution. b) UV-Vis spectrum of an empty quartz cuvette previouslyequilibrated with a $50 \mu \mathrm{M}$ MB solution. The cuvette was washed with a small amount of water and left dripping for $5 \mathrm{~min}$ following its treatment with MB. This spectrumrepresents the transmission UV-Vis spectrum of MB adsorbed on quartz. c) The same as b), but in this case a $1.0 \mathrm{mM} \mathrm{MB}$ solution was used. All spectra are normalized to the absorbance measured at $664 \mathrm{~nm}$. This transformation magnifies spectra b) and c) ca. 10-timescompared to spectrum a). The absorption peak of the MB monomer at $664 \mathrm{~nm}$ dominates spectra a) and b), and the peakat $612 \mathrm{~nm}$ is characteristic for the of the MB dimer in spectrumc).

Some of the kinetic experimentswere repeated using $50(\mathrm{~V} / \mathrm{V}) \%$ ethanol as the solvent for MB. We found that MB adsorbs on the quartz cuvette even from 1:1 ethanol-water. The kinetic curvesand the spectral change attributed to MB adsorptionfrom 1:1 ethanol are almost identical to those recorded in water. (Fig. S15inthe SI). The most obvious difference is the 
larger amplitude of absorbance change in 1:1 ethanol, which can be attributed to the ca. $10 \%$ increase of the molar absorbance of MB in ethanol. [31] The lack of any significant solvent effects means that the adsorption of $\mathrm{MB}$ on quartz isnot influenced by its solution phase dimerization, as this issuppressed inethanol. [32]It also follows that it is notthe dimer which promotes monolayer formation on the surface.

\section{Discussion}

4.1. General considerations. There is a small but reproducible absorbance decay after introducing an aqueous MB solution into a quartz cuvette (Fig. 1).In general, such an effect can be the consequence of several chemical or physical processes, many of which can be excluded on the basis of straightforward arguments as discussed in the next paragraphs.

When MB solutions of different concentrations are diluted to the same final concentration, the dimerization equilibrium of $\mathrm{MB}$ is shifted to the same final position. However, this process can be excluded as the source of the noted absorbance effect because the magnitude of the absorbance decay does not depend on the extent of dilution (Fig. S6) and was also observed when a dilute MB solution was added to a dry empty cuvette. This conclusion is corroborated by the kinetic profiles recorded in $50(\mathrm{~V} / \mathrm{V}) \%$ ethanol where the dimerization is suppressed(Fig. S15). The reproducibility of the experiments in pure water at pH 7 (Fig. S7) and in 50(V/V)\% ethanol excludes the possibility that some sort of impurity in the buffer solution causes the observed absorbance change. Redox reactions between MB and fortuitous contaminants were also excluded by considering that the process is reversible (Fig. S10). Thus, the only plausible explanation for the observations is the reversible adsorption of the dye on the quartzwall of the cuvette. $[13,14]$ The detrimental effect of hydrophobization of the surface on the adsorption clearly verifies that the surface needs to be hydrophilic for bonding MBefficiently (Fig. S12).

Our experimental observations indicate that protolytic equilibria involving the quartz surface and the stirring rate of the solutionhave profound effects on the adsorption process.Under acidic conditions, the silica surface and, to some extent, $\mathrm{MB}$ ( $\mathrm{p} K_{a}=1.69$ [1]) are protonated, thus, the attractive Coulomb interaction between them diminishes. This leads to limited adsorption of the dye.Basic $\mathrm{pH}$ enhances the negative surface charge of silica, thus, facilitates the adsorption(Fig. S8).In the absence of stirring, replenishment of MB at the 
quartz surface is maintained by a slow, rate limiting diffusion of the dye (Fig. S5). Stirring provides an efficient way for transporting $\mathrm{MB}$ to the surface and the rate of the adsorption becomes independent of stirring at sufficiently high stirring rates. In our case, the stirring rate was set at $1000 \mathrm{rpm}$ in order to avoid complications from diffusion processes. Under such conditions, the binding of MB to the surface is the actual rate limiting process.

4.2. Adsorption isotherm. The characteristic isothermfor the adsorption of MBon quartzwas constructed by plotting the amplitude of the absorbance change recorded in the kinetic experiments(Fig. 2) as a function of the MB concentration (c(MB)) as shown in Fig. 5.

Earlier studies, using the dipping method, indicated that MBadsorbs to silica only as a monolayer even from a $1.0 \mathrm{mM}$ solution. $[13,14]$ None of our experimental results suggest a deviation from monolayer adsorption, thus the isotherm measured on aquartz cuvette was evaluated accordingly. First, the isotherm was fitted to the Langmuir modelrestricting the adsorption of $\mathrm{MB}$ to one molecule per one active site on the surface. The extent of dimerization of $\mathrm{MB}$ in aqueous solution is smallin the applied concentration range, and negligible in the context of the model. The following equations describe the equilibrium, and account for the mass balances [33]:

$\mathrm{MB}+\mathrm{SS} \rightleftharpoons$

$K_{\mathrm{ads}}=\frac{\theta_{\mathrm{SMB}}}{[\mathrm{MB}] \theta_{\mathrm{S}}} \quad c=[\mathrm{MB}]+s \theta_{\mathrm{SMB}} / V \quad 1=\theta_{\mathrm{S}}+\theta_{\mathrm{SMB}}$

where MB is hydrated methylene blue, $\mathrm{S}$ represents an unoccupied site on the surface and SMB is the adsorbed dye occupying a site. $K_{\text {ads }}$ is the equilibrium constant of monolayer adsorption, $V$ is the volume of the solution in the cuvette. Thetotalconcentration of MB is $c$ and the number of moles of adsorption sites on the quartz cuvette is $s$. Square brackets denoteequilibrium concentrations. $\theta_{\mathrm{S}}$ and $\theta_{\mathrm{SMB}}$ representthe surface coverage offree and occupied sites, respectively. The absorbance change associated with the adsorption of MB on quartz $(\triangle A)$ is due to the lower apparent molar absorptivity of adsorbed MB compared to hydratedMB. On the basis of Beer's law, $\Delta A$ is given as follows (see the Supporting Information): 


$$
\begin{aligned}
& \Delta A=\varepsilon_{\mathrm{sol}} l_{\mathrm{sol}} c-\left(\varepsilon_{\mathrm{sol}} l_{\mathrm{sol}}[\mathrm{MB}]+\varepsilon_{\mathrm{ads}} l_{\mathrm{ads}}[\mathrm{SMB}]\right)=\left(\varepsilon_{\mathrm{sol}} l_{\mathrm{sol}}-\varepsilon_{\mathrm{ads}} l_{\mathrm{ads}}\right)[\mathrm{SMB}]= \\
&=E[\mathrm{SMB}]
\end{aligned}
$$

Here $\varepsilon_{\text {sol }}$ and $\varepsilon_{\text {ads }}$ are the molar absorbances of hydrated and adsorbed MB, respectively; while $l_{\text {sol }}$ and $l_{\text {ads }}$ are the optical pathlengths. $E$ is the difference between the molar absorptivity of hydrated and adsorbed MB. The molar absorbance of hydrated MB is well known and $l_{\text {sol }}=$ $1.0 \mathrm{~cm}$ in our experiments. In principle, $l_{\mathrm{ads}}$ could be calculated from the thickness of the adsorption layer on the surface, but the uncertainty associated with estimating the thickness makes any effort to separate $\varepsilon_{\mathrm{ads}}$ and $l_{\mathrm{ads}}$ pointless. According to earlier literature, the absorbance of the adsorbed MB drops to about $10 \%$ compared to that of MB in solution. [6,13] Our observations are also in line with such a difference. As shown in Fig. 4, the absorbance of a $50 \mu \mathrm{M}$ MB solution is about ten times stronger than that of the adsorbed layer on quartz.This is not unexpected because the edged orientation of adsorbed MB molecules on the surface decreases their effective cross section toward the light beam.Also, the light beam in the spectrophotometer hits only a minor fraction of the adsorbed molecules on the wall of the cuvette. Thus, we assume that $E$ is $90 \%$ of $\varepsilon_{\text {sol }} \times l_{\text {sol }}$ and include this parameter with a fixed value in the calculations: $E=3.6 \times 10^{4} \mathrm{M}^{-1} . \Delta A$ can be given by combining Eqs. 2 and 3 (see details in the Supporting Information):

$$
\begin{aligned}
\Delta A=\frac{E s \theta_{\mathrm{SMB}}}{V} & =E \frac{1}{2}\left\{\left(\frac{s}{V}+c+\frac{1}{K_{\mathrm{ads}}}\right)-\sqrt{\left.\left(\frac{s}{V}+c+\frac{1}{K_{\mathrm{ads}}}\right)^{2}-\frac{4 s c}{V}\right\} \approx}\right. \\
& \approx E \frac{K_{\mathrm{ads}} c}{1+K_{\mathrm{ads}} c} s / V
\end{aligned}
$$

As shown in Fig. 5, equation 4 fits the experimental data reasonably well. The non-linear least squares fitting routine yields the following estimates for the parameters: $K_{\text {ads }}=(1.5 \pm 0.5)$ $\times 10^{4} \mathrm{M}^{-1}$ and $s=(7.0 \pm 1.8) \mathrm{nmol}$ (equivalent to $3.5 \mu \mathrm{M} \mathrm{MB}$ in $2.0 \mathrm{~mL}$ solution).

The visible total reflection spectrum of adsorbed $\mathrm{MB}$ on quartz is reported to show a blue shift in the $600-700$ nmwavelength range. A similar blue shift is also associated withthe formation ofthe hydratedMB dimer. $[13,18]$ On the basis of these observations, Higgins et. al. proposed, that MB molecules adsorbedon quartz toform a monolayer of dimers. Although we found no blue shift in theUV-Vis spectrum of adsorbed MB in the studied concentration range (Fig. 4), we decided to test the validity of the adsorption modelpostulated by Higgins et. al.In this case, the Langmuir modelgives the equilibrium between solution phase and adsorbed 
dimers. When only the dimer adsorbs on the surface, and $c>>\left[(\mathrm{MB})_{2}\right]+\left[\mathrm{S}(\mathrm{MB})_{2}\right]$, the following equation and expressions apply (cf. the SI):

$$
\begin{aligned}
& 2 \mathrm{MB}\left(\Longrightarrow \quad(\mathrm{MB})_{2}+\mathrm{SS}(\mathrm{MB})_{2} \rightleftharpoons\right. \\
& K_{\mathrm{f}}=\frac{\left[(\mathrm{MB})_{2}\right]}{[\mathrm{MB}]^{2}} K_{\mathrm{ads} 2}=\frac{\theta_{\mathrm{SMB} 2}}{\left[\mathrm{MB}_{2}\right] \theta_{\mathrm{S}}} c=[\mathrm{MB}]+2\left[(\mathrm{MB})_{2}\right]+2 s \theta_{\mathrm{SMB} 2} / V \quad 1=\theta_{\mathrm{S}}+\theta_{\mathrm{SMB} 2} \\
& \Delta A=2 E \theta_{\mathrm{SMB} 2} s / V \\
& =2 E \frac{K_{\mathrm{ads} 2} K_{\mathrm{f}} c^{2}}{1+K_{\mathrm{ads} 2} K_{\mathrm{f}} c^{2}} s / V
\end{aligned}
$$

where $K_{\mathrm{f}}$ is the dimerization constant of hydrated $\mathrm{MB}$ and $K_{\mathrm{ads} 2}$ is the equilibrium constant describing monolayer adsorption of the MB dimer. Other symbols were defined earlier.The calculations were performed by either allowing parameters $K_{\text {fand }}$ Eto float or fixing their values at $10^{3.9} \mathrm{M}^{-1}$ and $3.6 \times 10^{4} \mathrm{M}^{-1}$, respectively. This had no effect on the goodness of the fit. The comparison of the experimental and fitted data (Fig. 5) clearly shows that the model is not suitable for the interpretation of the results. The characteristic sigmoid profile of the calculated curve is absent from the experimental data.

Adachi et al.refined the adsorption model by allowing the adsorption both the monomer and dimer $\mathrm{MB}$ on $\mathrm{WO}_{3}$ surface. This model also postulates that a dimer occupies two active sites on $\mathrm{WO}_{3}$ surface. [34] These authors reported ca. $80 \%$ surface dimerization at higher MB concentrations. We also testedAdachi's model on quartz, and found that the fitting results overlap with those obtained on the basis of Eq.4. These calculations predict that the surface dimerization constant is zero within experimental error,i.e.only the adsorption of the monomer form of MB occurs on quartz surface.

The results confirm that the quartz surface does not enhance the dimerization ofMB in the adsorption layer.This conclusion is also supported by the noted similarity between the $\mathrm{UV}-\mathrm{Vis}$ spectra of adsorbed and solution phase MB recorded after equilibrating the quartz surface with relatively low concentration MB solution (Fig. 4). 


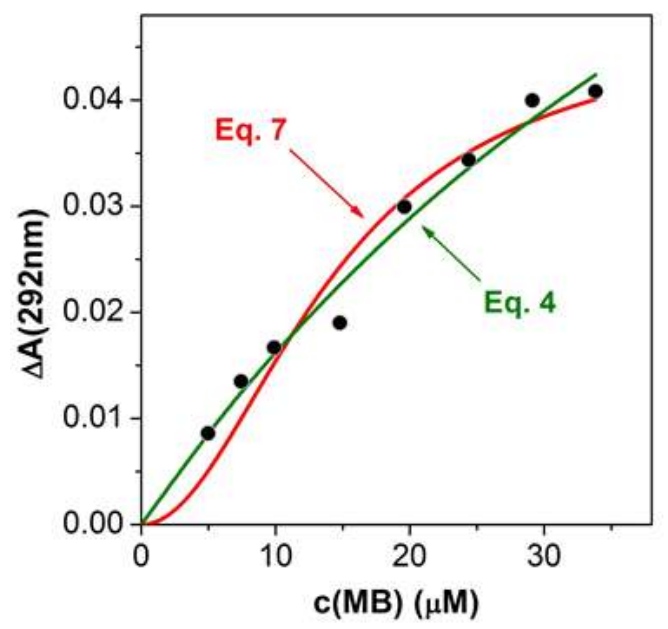

Figure 5. Experimental isotherm describing the adsorption of methylene blue (MB) on the surface of a quartz cuvette. Black points: difference between measured initial and equilibrium absorbance values(i.e., amplitude of spectral change, $\Delta A$ ) as a function of the analytical concentration of $\mathrm{MB}$ $(c(\mathrm{MB}))$. The corresponding experimental kinetic curves are shown inFig. 3. Green line: best nonlinear least squares fit to the Langmuirisotherm, assuming that only MB monomers adsorb to the surface (Eq. 4). Red line: best non-linear least squares fit assuming that only MB dimers adsorb to the surface (Eq. 7).

$\left(\mathrm{NaH}_{2} \mathrm{PO}_{4}+\mathrm{Na}_{2} \mathrm{HPO}_{4}\right)=50 \mathrm{mM} ; \mathrm{pH}=6.89 ; 25^{\circ} \mathrm{C} ; 1000$ rpm mixing.

4.3. The coverage of surface by methylene blue. The amount of adsorbed MB at the maximum surface coverage of the quartz cuvette is $s=(7.0 \pm 1.8) \mathrm{nmol}$. This value wasestimated on the basis of the Langmuirisotherm describing monolayer formation on the surface as discussed in the previous section. The volume of the MB solution is $2.0 \mathrm{~cm}^{3}$ and the surface of the cuvette covered by this solution is $9.0 \mathrm{~cm}^{2}$. (When a cuvette with $1.000 \mathrm{~cm}$ optical pathlength is used, the solution is in contact with the vertical walls up to $2.0 \mathrm{~cm}$ and the $1.0 \mathrm{~cm}^{2}$ bottom part of the cuvette.) This translates into $7.8 \times 10^{-10} \mathrm{~mol}$ adsorbed MB per $\mathrm{cm}^{2}$ quartz. Therefore, the calculated area per adsorbed MB molecule is $21 \AA^{2}\left(21 \times 10^{-20}\right.$ $\mathrm{m}^{2}$ ). This value is in good agreement with the area requirement of MB, i.e., $22-24 \AA^{2}$, measured previously under conditions when a monolayer forms, and $\mathrm{MB}$ molecules bind roughly perpendicularly to the surface. $[4,34]$

4.4 Effect of MB concentration on theadsorption rate. The available amount of monolayer adsorption sites was $7.0 \mathrm{nmol}$ on the cuvetteand the amount of MB present in solution was 60 nmol $(2.0 \mathrm{~mL}$ of a $30 \mu \mathrm{M}$ solution) at most. The small difference in the amount of available sites and MB predicts that the outer hydration sphere of the sites was never saturated with MB. This is in agreement with the proposal that the adsorption of MBisfaster with increasing 
MB concentration (cf. Fig. 2). Also, the rate of adsorption did not level off, as would be expected if it were controlled only by the amount of dye in theouter hydration sphere of quartz.On the contrary, $\mathrm{MB}$ was rapidly adsorbed and effective mixing was needed to replenish it near the surface. Without mixing, the replenishment of the dye near the surface was limited by its diffusion, thus the overall adsorption process was slow ( $c f$. Fig. S5). When mixing was introduced, the concentration gradient vanished, and adsorption was accelerated.

4.5. Kinetics and mechanism of adsorption. As discussed in the Results section, the kinetic curves recorded following the introduction of $\mathrm{MB}$ solutions into the quartz cuvette were generally biphasic, regardless of the MB concentrationapplied(Fig. 2). First, we have to emphasize that the shift of the solution phase dimerization equilibrium of MB during in situ dilution does not have any kinetic effect under our experimental conditions. The shape of the observed kinetic curves is invariant to the ratio of in situ dilution. If solution phase dimerization were slow and had significant contribution to the measured absorbance decay, the kinetics should depend on the extent of dilution, as more dimer should dissociate to monomer when diluting a more concentrated MB solution to the same final concentration.

A kinetic model was developed to describe the adsorption kineticson the basis of the following considerations: $i$ ) the absorbance change is solely caused by the adsorption of MB on quartz;ii) in line with the analysis of the adsorption isotherm,only monolayer adsorption of the monomer occurs;iii) the observed biphasic kinetics may be consistent with dimerization on the surface, butthe molar fraction of the adsorbed dimerscannotexceed that of hydrated dimers, as discussedin connection with the transmission UV-Vis spectrum of adsorbed MB (Fig.4);iv) on a longer time scale, the isomerization of the binding of MB can also be expected. [12]

The simplest model to give biphasic kinetics and include the adsorption of both dimers and monomers is practically the consecutive adsorption of two MB molecules to the same surface site.The same kinetic model is proposed by Qi et. al. to interpret optical waveguide spectroscopic results studying the adsorption of MB onto glass surface. [35]However, we confirmed that this modelis inadequate to describe our experimental kinetic data.The model,together with the detailed description of the fitting procedure [36] and its results, is given in the Supporting Information( $c f$. Scheme S1).

We propose an alternative kinetic model,which postulates relatively fast reversible adsorption of $\mathrm{MB}$ on the surface in the first step and aslowerisomerization of the binding of theadsorbed species, which is also a reversible reaction. The kinetic model is presented in 
Scheme 1. It is important to note that this model assumesthe existence of two different binding modeson quartz.The model with two binding modes is mathematically equivalent with themodel featuring two binding sites.Thus, the two models are kinetically indistinguishable.The experimental kinetic curves (Fig. 2) were fitted simultaneously to the model of Scheme 1, as detailed in the SI. The monolayer capacity of quartz was set to be $7.0 \mathrm{nmol}$. The resulting fit is good at every initial concentration as shown in Fig. 2. Estimated rate constants are given in Scheme 1.

$$
\begin{array}{lcc}
\mathrm{R} 1) & \mathbf{M B}+\mathbf{S} \rightarrow \mathbf{S M B} & k_{1}=(1.36 \pm 0.03) \times 10^{-3} \mu \mathrm{M}^{-1} \mathrm{~s}^{-1} \\
\mathrm{R} 2) & \mathbf{S M B} \rightarrow \mathbf{M B}+\mathbf{S} & k_{2}=(1.29 \pm 0.03) \times 10^{-1} \mathrm{~s}^{-1} \\
\mathrm{R} 3) & \mathbf{S M B} \rightarrow \mathbf{S M B} & k_{3}=(5.3 \pm 0.4) \times 10^{-3} \mathrm{~s}^{-1} \\
\mathrm{R} 4) & \mathbf{S M B} \rightarrow \mathbf{S M B} & k_{4}=(1.46 \pm 0.06) \times 10^{-2} \mathrm{~s}^{-1} \\
\frac{d[\mathrm{MB}]}{d t}=-k_{1}[\mathrm{MB}] \theta_{\mathrm{S}} s / V+k_{2} \theta_{\mathrm{SMB}} S / V \\
\frac{d \theta_{\mathrm{SMB}}}{d t}=+k_{1}[\mathrm{MB}] \theta_{\mathrm{S}}-k_{2} \theta_{\mathrm{SMB}}-k_{3} \theta_{\mathrm{SMB}}+k_{4} \theta_{\mathrm{SMBI}} \\
\frac{d \theta_{\mathrm{SMBI}}}{d t}=+k_{3} \theta_{\mathrm{SMB}}-k_{4} \theta_{\mathrm{SMBI}} \\
\frac{d \theta_{\mathrm{SMB}}}{d t}=-k_{1}[\mathrm{MB}] \theta_{\mathrm{S}}+k_{2} \theta_{\mathrm{SMB}}
\end{array}
$$

Scheme 1. Kinetic model detailing the adsorption of a methylene blue molecule to a surface site on quartz. The first process (reactions R1 and R2) is the reversible monolayer adsorption of the dye, and the second process (reactions R3 and R4) is an isomerization in the mode of binding on the surface. Square brackets indicate time-dependent actual concentrations, and $k_{\mathrm{i}}$ represent rate constants. $\theta$ is timedependent surface coverage. The analysis of time resolved spectral data based on this kinetic model gives good results. Further details are given in the text and in Fig. 6.

The observed spectral changes canalso be successfully reconstructed by using the kinetic model of Scheme 1 and the rate constants estimated with the global leastsquares fit. The calculated spectra of MB present in different binding modes on quartz are reasonable. 
The calculation, as expected, is not sensitive to $c(\mathrm{MB})$, as seen in Fig. 6. It is interesting to note that no blue shift can be observed in these reconstructed spectra, which is in good agreement with the measured transmission UV-Vis spectra of adsorbed MB (Fig. 4). The assumption that themolar absorbance of hydrated MB decreases by ca. $90 \%$ upon adsorption on quartz is also strengthened by these results.

Thus, we can conclude that the kinetic model proposed in Scheme 1 is adequate to describe the kinetics of adsorption of MB from dilute solution onto quartz.

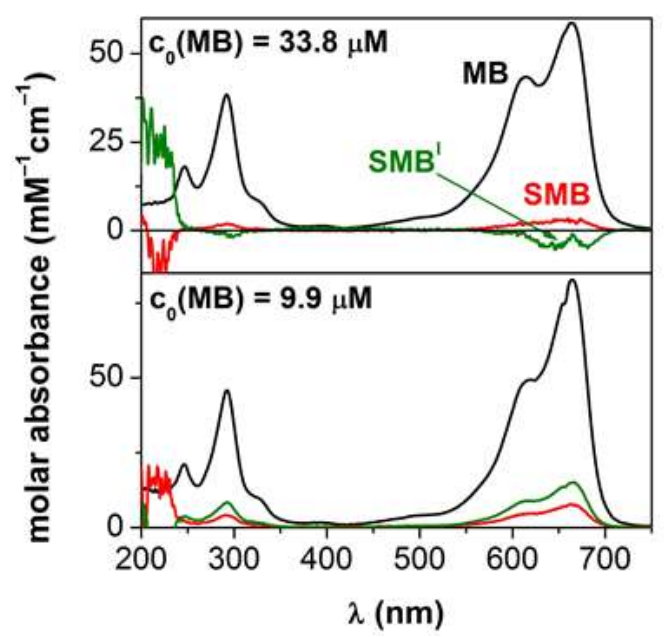

Figure 6. Calculated UV-Vis spectra of hydrated MB and MB monomers adsorbed on quartz surface in different modes of binding. SMB is the primary product of adsorption, and $\mathrm{SMB}^{\mathrm{I}}$ forms on a longer time scale by the isomerization of binding. The calculation was based on the kinetic model of Scheme lusing estimated parameters from simultaneously fitting all experimental kinetic curves of Fig. 2. $\mathrm{c}\left(\mathrm{NaH}_{2} \mathrm{PO}_{4}+\mathrm{Na}_{2} \mathrm{HPO}_{4}\right)=50 \mathrm{mM} ; \mathrm{pH}=6.89 ; 25^{\circ} \mathrm{C} ; 1000 \mathrm{rpm}$ mixing.

\section{Conclusions}

The adsorption isotherm of MB on quartzwas measured in the concentration range $c(\mathrm{MB})=5-34 \mu \mathrm{M}$.It was shown that the isotherm is not consistent with the exclusive binding of $\mathrm{MB}$ dimers to quartz surface, whichwasproposed in the literature. $[13,14,18]$ Furthermore, the transmission UV-Vis spectrum of adsorbed MB does not show any blue shift when $10-50 \mu \mathrm{M}$ MB solutions are used for equilibration.

Under controlled conditions, we were able to follow the kinetics of the relatively fast adsorption of $\mathrm{MB}$ from aqueous solution onto the surface of a quartz cuvette by simply 
monitoring the absorbance change.The adsorption process is kinetically first order with respect to $\mathrm{MB}$. This observation confirms that the formation of $\mathrm{MB}$ dimer does not contribute to the overall adsorption process and corroborate our conclusion that dimerization of $\mathrm{MB}$ is not favored on quartz surface.A kinetic model is postulated to describe the mechanism of the adsorption process. The simplest model that reliably interprets the multi-wavelength kinetic resultsconsists of two steps: $i$ ) the reversible adsorption of $\mathrm{MB}$ on quartz surface; $i i)$ an isomerization in binding mode.

Careful evaluation of the kinetic and thermodynamic data serves straightforward evidence thatquartz surface does not promote binding of the MB dimer over the monomer. Both the thermodynamic and the kinetic results suggest, that afterthe introduction of $\mathrm{MB}$ solution into a cuvette, a monolayer is formed on quartz from single MB molecules which differ from each other only in the mode of binding.

\section{Acknowledgements}

The assistance of Réka Tóth (University of Debrecen) with some experiments is greatly acknowledged. The authors thank the Hungarian Science Foundation (OTKA: NK-105156) and the University of Debrecen (RH/751/2015) for financial support.The research was supported by the EU and co-financed by the European Social Fund under the project ENVIKUT (TÁMOP-4.2.2.A-11/1/KONV-2012-0043).

\section{Appendix A. Supplementary material}

UV-Vis spectroscopic data on the dimerization of methylene blue. Detailed kinetic and spectroscopic information in the form of additional figures and tables on the adsorption of methylene blue. This material can be found in electronic Supporting Information. 


\section{References}

[1] Ghosh AK. Study of the self-association of methylene blue from protonation equilibriums. J Am Chem Soc 1970; 92: 6415-8.

[2] Mukerjee P, Ghosh AK. Thermodynamic aspects of the self-association and hydrophobic bonding of methylene blue. Model system for stacking interactions. J Am Chem Soc 1970, 92: 6419-24.

[3] Patil K, Pawar R, Talap P. Self-aggregation of methylene blue in aqueous medium and aqueous solutions of $\mathrm{Bu}_{4} \mathrm{NBr}$ and urea. Phys Chem Chem Phys 2000; 2: 4313-7.

[4] Allingham MM, Cullen JM, Giles CH, Jain SK, Woods JS, Adsorption at inorganic surfaces. II. Adsorption of dyes and related compounds by silica. J Appl Chem 1958; 8: 10816.

[5] Rafatullah M, Sulaiman O, Hashim R, Ahmad A. Adsorption of methylene blue on lowcost adsorbents: a review. J Hazard Mater 2010; 177: 70-80.

[6] Yan Y, Zhang M, Gong K, Su L, Guo Z, Mao L. Adsorption of methylene blue dye onto carbon nanotubes: a route to an electrochemically functional nanostructure and its layer-bylayer assembled nanocomposite. Chem Mater 2005; 17: 3457-63.

[7] Zhang W, Zhou C, Zhou W, Lei A, Zhang Q, Wan Q, Zou B. Fast and considerable adsorption of methylene blue dye onto graphene oxide. Bull Environ Contam Toxicol 2011; 87: $86-90$.

[8] Jockusch S, Turro NJ, Tomalia, DA. Aggregation of methylene blue adsorbed on starburst dendrimers. Macromolecules 1995; 28: 7416-8.

[9] Bujdák, J, Komadel, P. Interaction of methylene blue with reduced charge montmorillonite.J Phys Chem B 1997; 101: 9065-8.

[10] Chen, G, Pan, J, Han, B, Yan, H. Adsorption of methylene blue on montmorillonite.J Dispersion Sci Technol 1999; 20: 1179-87.

[11] Vargas AM, Cazetta AL, Kunita MH, Silva TL, Almeida VC. Adsorption of methylene blue on activated carbon produced from flamboyant pods (Delonix regia): Study of adsorption isotherms and kinetic models.Chem Eng J 2011; 168: 722-30.

[12] Garoff S, Stephens RB, Hanson CD, Sorenson GK. Surface interactions of adsorbed molecules as probed by their optical properties.Opt Commun 1982; 41: 257-62.

[13] Higgins DA, Byerly SK, Abrams MB, Corn RM. Second harmonic generation studies of methylene blue orientation at silica surfaces.J Phys Chem 1991; 95: 6984-90.

[14] Tsunoda KI, Umemura T, Ueno H, Okuno E, Akaiwa H. Adsorption of methylene blue onto silylated silica surfaces, studied using visible attenuated total reflection spectroscopy with a slab optical waveguide.Appl Spectrosc 2003; 57: 1273-7. 
[15] Yao H, Li N, Xu S, Xu JZ, Zhu JJ, Chen HY. Electrochemical study of a new methylene blue/silicon oxide nanocomposition mediator and its application for stable biosensor of hydrogen peroxide.Biosens Bioelectron 2005; 21: 372-7.

[16] Deboux BC, Lewis E, Scully PJ, Edwards R. A novel technique for optical fiber pH sensing based on methylene blue adsorption.J Lightwave Technol 1995; 13: 1407-14.

[17] Chakrabarti S, Dutta BK. On the adsorption and diffusion of methylene blue in glass fibers.J Colloid Interface Sci 2005; 286: 807-11.

[18] Bergmann K, O'konski CT. A spectroscopic study of methylene blue monomer dimer and complexes with montmorillonite.J Phys Chem 1963; 67: 2169-77.

[19] Beissinger RL, Leonard EF. Plasma protein adsorption and desorption rates on quartz: approach to multi-component systems. ASAIO Journal 1981; 27: 225-30.

[20] Hu J, Yang D, Kang Q, Shen D. Estimation the kinetics parameters for non-specific adsorption of fibrinogen on quartz surface from the response of an electrode-separated piezoelectric sensor.Sens Actuators B 2003; 96: 390-8.

[21] Mifflin AL, Gerth KA, Geiger FM. Kinetics of chromate adsorption and desorption at fused quartz/water interfaces studied by second harmonic generation.J Phys Chem A 2003; 107: 9620-7.

[22] Covington AK, Bates RG, Durst RA. Definition of $\mathrm{pH}$ scales standard reference values measurement of $\mathrm{pH}$ and related terminology. Pure Appl Chem 1983; 55: 1467-76.

[23] Gombár M, Józsa É, Braun M, Ösz K. Construction of a photochemical reactor combining a CCD spectrophotometer and a LED radiation source.Photochem Photobiol Sci 2012; 11: 1592-5.

[24] Peintler G: ZiTa: A comprehensive program package for fitting parameters of chemical reaction mechanisms Version 41 Institute of Chemistry JATE Szeged Hungary 1997 The first use of this program was described in:

Peintler G, Nagypal I, Epstein IR. Systematic design of chemical oscillators 60 Kinetics and mechanism of the reaction between chlorite ion and hypochlorous acid.J Phys Chem 1990; 94: 2954-8.

[25] Hindmarsh AC: GEAR: Ordinary Differential Equation SolVer ReV 2. Lawrence Livermore Laboratory 1972.

[26] Gaspar A, Gomez FA. Application of surface plasmon resonance spectroscopy for adsorption studies of different types of components on poly(dimethylsiloxane). Anal Chim Acta $2013 ; 777: 72-7$.

[27] Santos JH, Matsuda N, Qi ZM, Yoshida T, Takatsu A, Kato K. Experimental evidence of the reversibility of the first stage of protein adsorption at a hydrophobic quartz surface near the isoelectric point.Surf Interface Anal 2003; 35: 432-436. 
[28] Peintler G, Nagypál I, Jancsó A, Epstein IR, Kustin K. Extracting experimental information from large matrixes 1 . A new algorithm for the application of matrix rank analysis.J Phys Chem A 1997; 101: 8013-20.

[29] Kalmár J, Ellis SB, Ashby MT, Halterman RL. Kinetics of formation of the host-guest complex of a viologen with cucurbit[7]uril. Org Lett 2012; 14: 3248-51.

[30] Szabó M, Baranyai Z, Somsák L, Fábián I. The decomposition of N-chloroglycine in alkaline aqueous solution: kinetics and mechanism.Chem Res Toxicol 2015; 28: 1282-91.

[31] Tafulo PAR, Queirós RB, González-Aguilar G. On the "concentration-driven" methylene blue dimerization.Spectrochim Acta Mol Biomol Spectrosc 2009; 73: 295-300.

[32] Fornili SL, Sgroi G, Izzo V. Effects of solvent on stacking interactions A spectrophotometric study of methylene blue dimerization in aqueous solutions of some monohydric alcohols.J Chem Soc Faraday Trans 1983; 79: 1085-90.

[33] Beck MT, Nagypál I. Chemistry of Complex Equilibria.Budapest: Akadémiai Kiadó; 1990.

[34] Adachi K, Mita T, Yamate T, Yamazaki S, Takechi H, Watarai H. Controllable adsorption and ideal $\mathrm{H}$-aggregation behaviors of phenothiazine dyes on the tungsten oxide nanocolloid surface. Langmuir 2009; 26: 117-25.

[35] Qi ZM, Matsuda N, Takatsu A, Kato K. In situ investigation of coadsorption of myoglobin and methylene blue to hydrophilic glass by broadband time-resolved optical waveguide spectroscopy. Langmuir 2004; 20: 778-84.

[36] Lente G. Deterministic kinetics in chemistry and systems biology: The dynamics of complex reaction networks. New York: Springer; 2015. 